Neurosurg Focus 20 (4):E19, 2006

\title{
Treatment options for glioblastoma
}

\author{
Marc C. Chamberlain, M.D. \\ Department of Interdisciplinary Oncology, Moffitt Cancer Center and Research Institute, \\ Tampa, Florida
}

\begin{abstract}
$\checkmark$ Following the seminal trial conducted by the European Organisation for Research and Treatment of Cancer (EORTC) and the National Cancer Institute of Canada (NCIC), concurrent temozolomide and radiotherapy has become the new standard of care for patients with newly diagnosed glioblastoma multiforme (GBM). Investigation of emerging therapies (which are now used as salvage therapy) such as small-molecule inhibitors (for example, epidermal growth factor receptor inhibitors) and convection-enhanced delivery (CED) of targeted toxins (for example, interleukin-13/pseudomonas exotoxin) is likely to build on the EORTC/NCIC treatment platform and will, it is hoped, improve survival rates in patients with GBM. The majority of adjuvant Phase I and II trials being conducted by the brain tumor consortia are based on the EORTC/NCIC treatment platform and have added a targeted therapy in an effort to find a promising synergistic treatment. Furthermore, researchers in the consortia are continuing to explore treatments for recurrent GBM, not otherwise eligible for local therapies, such as CED. The treatments under study include novel cytotoxic chemotherapy as well as small-molecule inhibitors; these are being assessed in a variety of Phase I or II trials.
\end{abstract}

\section{KEY WORDS - glioblastoma multiforme - adjuvant treatment $\quad$ salvage treatment $\bullet$ cytotoxic chemotherapy $\quad$ convection-enhanced delivery $\bullet$ small-molecule inhibitor}

A CCORDING to World Health Organization estimates, there are 100 different types of brain tumor. Furthermore, the data obtained for the Surveillance, Epidemiology, and End Results Program yielded an estimate of 18,500 new cases of primary brain tumors in $2005 .{ }^{61}$ Of these, $50 \%$ were glial and $50 \%$ of all gliomas were GBMs. Glioblastomas multiforme are biologically aggressive tumors that present unique treatment challenges due to the following characteristics: 1) localization of tumors in the brain; 2) intrinsic resistance of these lesions to conventional therapy; 3) limited capacity of the brain to repair itself; 4) the spread of malignant cells into brain parenchyma; 5) the variably disrupted blood-brain barrier complicating drug delivery; 6) tumor capillary leakage, with resultant peritumoral edema and intracranial hypertension; 7) the limited response to therapy; and 8) the neurotoxicity of treatments directed at gliomas.

Based on early trials by BTSG and RTOG investigators, treatment of newly diagnosed GBMs has evolved to include maximum safe extent of resection (as enunciated in

\footnotetext{
Abbreviations used in this paper: $\mathrm{BCNU}=1,3$-bis(2-chloroethyl)1-nitrosourea; BTSG = Brain Tumor Study Group; CED = convection-enhanced delivery; EGFR = epidermal growth factor receptor; EORTC = European Organisation for Research and Treatment of Cancer; $\mathrm{GBM}=$ glioblastoma multiforme; $\mathrm{HDAC}=$ histone deacetylase; $\mathrm{IL}=$ interleukin; MGMT $=\mathrm{O}^{6}$ methylguanine-DNA methyltransferase; NCIC $=$ National Cancer Institute of Canada; PI3K = phosphatidylinositol 3-kinase; poly-ICLC = polyinosinic-polycytidylic acid stabilized with polylysine and carboxymethylcellulose; RTOG = Radiation Therapy Oncology Group; SAHA = suberoylanilide hydroxamic acid; TMZ = temozolomide.
}

the National Comprehensive Cancer Network central nervous system guidelines), conventionally fractionated external-beam radiotherapy to the tumor (as defined by the RTOG), and the administration (or not) of alkylator chemotherapy (most often nitrosoureas such as BCNU). ${ }^{22,76,77}$ The value of near-total or complete resection for GBM continues to be debated, primarily because no prospective trial has been conducted to assess the extent of resection as a primary outcome measure. ${ }^{16,43,47,49,52,66,67}$ A single metaanalysis, multiple single-institution retrospective studies, and data from the BTSG all support the value of large-volume resection in adults with GBM; however, other opinions exist.

The value of adjuvant chemotherapy has been debated and remains controversial despite prior metaanalyses by Fine, et al., ${ }^{25}$ and more recently by Stewart. ${ }^{70}$ Their studies demonstrated modest improvement in 1- and 2-year survival rates (5-6 and 4-5\%, respectively) with the inclusion of adjuvant chemotherapy. Last year, and during the conduct of the Glioma Outcomes Project (a project comprising 560 patients with newly diagnosed gliomas that preceded the EORTC/NCIC publication), in which 58 community and university centers were involved, the following pattern of care for adults with newly diagnosed gliomas was seen. ${ }^{16,52}$ All patients underwent surgery; $87 \%$ received radiotherapy; $88 \%$ received anticonvulsant therapy (the majority not in accordance with the American Academy of Neurology guidelines); $54 \%$ received chemotherapy; $29 \%$ used alternative medicines; and $15 \%$ enrolled in clinical trials. ${ }^{16,32}$

The preliminary reports of Stupp and colleagues ${ }^{71,72}$ and the recently published randomized European and Canadian trial have substantially altered the algorithm for initial treat- 
ment of GBM. These studies clearly demonstrated a benefit for chemotherapy (that is, TMZ) in the initial treatment of patients with GBM by showing an improvement in median (14.6 compared with 12 months) and 2-year survival (27 compared with 10\%) in patients receiving or not receiving TMZ. Consequently, this treatment regimen (TMZ given concurrently with radiotherapy, followed by six monthly cycles of TMZ) has become the new standard of care for patients with newly diagnosed GBM.

\section{Adjuvant Therapy}

The EORTC/NCIC study enrolled 573 patients in a randomized multicenter Phase III trial to compare radiotherapy alone with TMZ plus radiotherapy, followed by 6 months of postradiotherapy TMZ. ${ }^{72}$ Chemotherapy was given concurrently with conventional external-beam radiotherapy for 42 consecutive days (single daily dose $75 \mathrm{mg} / \mathrm{m}^{2}$ ), beginning with the first day of radiation. Radiotherapy was administered for 30 to 34 treatment days as a single 180- to 200-cGy fraction per day, to yield a total dose of 59.4 to $61.2 \mathrm{~Gy}$. The brain treatment volume was as defined by the EORTC and RTOG guidelines. ${ }^{2}$

Two to 3 weeks after the conclusion of concurrent chemo- and radiotherapy, patients were reevaluated with magnetic resonance imaging of the brain. If they were clinically and radiographically stable or had improved, patients were then treated with TMZ every 4 weeks (single daily dose 200 $\mathrm{mg} / \mathrm{m}^{2}$ for 5 consecutive days) for 6 months. Subsequent clinical and laboratory evaluations were completed monthly, and neuroradiographic evaluations were performed every other month.

As a study correlative, MGMT expression was evaluated in approximately 54\% of all patients (307 individuals); however, studies in only $206(36 \%)$ patients were informative with respect to MGMT expression..$^{38}$ The MGMT analysis (by promoter methylation status) is relevant because previous studies have indicated that MGMT is the predominant repair enzyme of alkylator-based chemotherapy-induced DNA injury and that gene silencing by MGMT (accomplished by promoter methylation) is correlated with improved response and survival rates. ${ }^{40}$

Outcome was significantly improved in patients treated with TMZ (2-year survival rate 27 compared with $10 \%$, and 2-year progression-free survival 11 compared with $2 \%$ ) and additionally in patients with methylated MGMT promoter who were treated with TMZ (median survival duration 21.7 compared with 15.3 months). Notably, in patients with unmethylated MGMT promoter, no difference was seen between those treated with TMZ and those treated with radiotherapy only (median survival 12.7 compared with 11.8 months).

Recently, an analysis of a secondary endpoint of the EORTC/NCIC trial, that is, health-related quality of life, revealed no negative effect on this endpoint with the addition of TMZ to radiotherapy. ${ }^{73}$ These results demonstrate that the addition of TMZ during and after radiotherapy for patients with newly diagnosed GBM both significantly improves survival and has no negative effect on quality of life. The results of the EORTC/NCIC study have several profound implications. First, the treatment protocol is now considered the new standard of care despite the lack of a confirmatory study (see later discussion). Second, there is an implication that TMZ may be an effective radiosensitiz- er, accounting in part for the improvement in overall survival. Nevertheless, this hypothesis has not been confirmed in an appropriately designed clinical trial. Third, postradiation therapy with TMZ was limited to 6 months, a duration of treatment $50 \%$ shorter than that customarily given in the US, where alkylator-based chemotherapy is more often given for 1 year. Fourth, the study results apply to the population treated (that is, patients $<70$ years old) and therefore do not clarify how best to treat the large group of elderly patients with GBM (estimated at $20 \%$ of all patients with GBM; see later discussion). In addition, the EORTC/NCIC study was confined to patients with GBM, and its results should not be extrapolated to other malignant gliomas such as anaplastic astrocytoma. Finally, the correlative studies support the suggestion that patients with GBM may be stratified into two similarly sized groups composed of individuals with or without gene silencing by MGMT.

The expression of MGMT had prognostic value, predicting a less favorable outcome (12.2-month median survival duration compared with 18.2 months), and furthermore, there was a low likelihood of benefit from alkylator-based chemotherapy. The best outcome was seen in patients without MGMT expression who were treated with TMZ; in this group the median survival duration was 21.7 months and the 2-year survival rate was $46 \%$. Nevertheless, it is difficult to know how best to treat patients in whom a poor response to alkylators (that is, with MGMT expression) can be presumed, because at present there are no chemotherapy alternatives to offer such patients. This aspect of patient stratification is likely to emerge as MGMT analysis becomes a standard part of patient care and tumor analysis, as is already common at several neurooncology centers.

Of considerable importance are three randomized studies with negative results that have broad implications for the care of patients with GBM. The first, initially a pilot study at The Johns Hopkins University that was subsequently evaluated in a randomized Phase III trial by the Eastern Cooperative Oncology Group, was an evaluation of neoadjuvant chemotherapy (3-day continuous infusions of cisplatin and BCNU) in adults with newly diagnosed GBMs. ${ }^{34,36}$ In this study, 223 patients were randomized either to radiotherapy and BCNU (administered during radiotherapy and every 6-8 weeks for a total of six cycles, which at the time was considered standard therapy) or to radiotherapy and neoadjuvant chemotherapy (to a maximum of three cycles). No difference in the median survival time was seen between patients in the two arms of the study (11 compared with 11.2 months). Several conclusions are relevant based on this study. First, the role for neoadjuvant chemotherapy in adults with GBM is of unproven and uncertain benefit. Second, response rates to preradiotherapy chemotherapy do not appear to translate into improved outcomes in patients with GBM. Third, the value of platinoid salt-based chemotherapy in the adjuvant treatment of GBM is as yet of uncertain benefit.

In the second study with negative findings, an RTOG trial, 203 newly diagnosed patients were randomized to receive radiotherapy with or without single-fraction stereotactically administered radiotherapy (gamma knife surgery). ${ }^{68}$ Gamma knife surgery was administered before conventional radiotherapy. No difference in outcome was seen (median survival time 13.5 compared with 13.6 months), and this supports the suggestion that boost radiotherapy does not 
benefit adult patients with GBM when administered in the adjuvant setting.

The results of this trial recapitulate those seen in an earlier BTSG investigation in which adjuvant brachytherapy was used for newly diagnosed GBM, where again no survival benefit was seen..$^{23,24,50,65}$ Therefore, these two randomized trials provide Level 1 evidence that additional radiotherapy beyond that administered conventionally and by external beam not only adds cost to these patients' care, but more importantly is of no proven benefit. In addition, a recent small Phase II trial (34 patients) of conformal radiotherapy in which a radiation dose of 90 Gy was used showed no improvement in either median or 2-year survival rates. ${ }^{15}$ The site of GBM recurrence after treatment failure was similar to that in other radiotherapy trials, with $78 \%$ central, $13 \%$ in-field, and $9 \%$ marginal field failures. Novel radiosensitizing agents such as efaproxiral sodium (RSR-13) and motexafin gadolinium continue to be explored in the adjuvant treatment of GBM, but at present their use is restricted to investigational trials. ${ }^{44,46}$

In the third study, another randomized Phase III trial, maximum safe resection (the median extent of resection was $\geq 90 \%$ ) followed by radiotherapy was compared with the same treatment that also included implantation of Gliadel wafers. ${ }^{79}$ In this study, 240 adults with newly diagnosed GBM were compared, and although a 2.4-month difference in survival duration favoring the Gliadel arm was seen initially (despite the fact that no survival benefit was seen at the 18-month interval), subsequent analysis that excluded a small population of anaplastic gliomas in which histological features of GBM were absent resulted in no difference in outcome (that is, similar median and 18-month survival rates). Therefore, Gliadel placement at the time of initial resection in patients with newly diagnosed GBM is currently of unproven value.

Elderly patients with GBM are a relatively understudied subgroup, despite the fact that they constitute a significant proportion of all patients with GBM $(\sim 20 \%)$. According to the Central Brain Tumor Registry in the US, 1- and 2-year survival in patients with GBM who are 65 years of age or older is 13.3 and $2.1 \%$, respectively. Furthermore, results of the RTOG recursive portioning analysis support the suggestion that the majority of elderly patients with GBM will be categorized as Class 6 and will have an expected median survival period of 4 months. ${ }^{20}$

Despite the paucity of studies, several strategies have been offered to treat this patient population..$^{10}$ In elderly patients with otherwise apparently excellent health and performance status, the EORTC/NCIC treatment is implemented. Analysis of the EORTC/NCIC data by age strata demonstrated no difference in outcome in patients who were 50 to 59 compared with those 60 to 69 years of age. ${ }^{71}$ Alternatively, other investigators have recommended accelerated hypofractionated radiotherapy ( $40 \mathrm{~Gy}$ in 15 fractions) in patients with impaired performance status. ${ }^{62}$ Another alternative approach has been to treat elderly patients with primary chemotherapy (TMZ given on a schedule of 5 consecutive days every 4 weeks) with deferred radiotherapy. ${ }^{31}$ Several cooperative groups are designing trials to address how best to treat elderly patients with newly diagnosed GBM.

\section{Salvage Therapy}

Two different, novel strategies have evolved for treating patients with recurrent GBM. In the first, direct regional administration of protein toxins is accomplished through stereotactically implanted catheters by using CED., , 17,23,45,51,55 In the second strategy, targeted therapies with small-molecule inhibitors of cell cycle signaling that are believed to be biologically relevant to the glioma phenotype are used with or without cytotoxic chemotherapy (Appendices 1 and 2)., $18,21,26,29,53,54,56,60$

Several intratumoral drug delivery systems have been or are in clinical trials (Appendix 3). Interstitial diffusionbased drug delivery systems such as controlled-release polymer implants (that is, Gliadel wafers) are limited by a small volume of distribution within the tumor and the brain surrounding it, and by high and heterogeneous drug concentrations that may be subtherapeutic or toxic. ${ }^{11,12,35}$ In contrast, CED can deliver drugs over a large brain volume and at relatively uniform concentrations by high-flow microinfusion that generates a positive pressure gradient, propelling the drug through the extracellular matrix. The CED procedure at present uses a targeted therapy (typically a protein ligand that binds to an overexpressed receptor specific to glioma cells; that is, IL-13 or transferrin) linked to a bacterial toxin (either pseudomonas or diphtheria toxin; Appendix 4). ${ }^{5,17,23,45,51,55}$ The rationale for CED is that it is a cytotoxic therapy that works independently of apoptotic factors, is not cell cycle-specific, functions in a hypoxic environment, functions independently of cell signaling pathways, is not subject to drug efflux mechanisms, and does not induce drug resistance. ${ }^{23,45}$

Two approaches in which CED is used have been pursued clinically. In one of them, the tumor is resected and catheters are implanted in the brain surrounding the resected tumor cavity (an approach used in the NeoPharm PRECISE trial [NeoPharm, Inc., Lake Forest, IL]). In the other, catheters are placed directly into the tumor (a method of delivery used in the TransMID trial [Xenova Biomedix Pharmaceuticals, Inc., Slough, Berkshire, United Kingdom). However, CED is limited to 1- to 4-cm supratentorial tumors not involving the midline, and in the NeoPharm PRECISE trial, to surgically resectable tumors. Randomized Phase III trials using both methodologies are being completed, which will ultimately define the use of CED in future treatment protocols. Another local therapy that is intracavitary, however, is now in clinical trials and uses the novel agent chlorotoxin ligated to iodine-131 (the drug is called TM-601). ${ }^{39}$ Patients eligible for this Phase II trial are to undergo repeated resection and are required to have noncerebrospinal fluid-communicating surgical cavities in which a reservoir system is implanted. Subsequently, patients are treated via a subgaleal reservoir with direct instillation of TM-601.

The molecular biology of gliomas has provided new insights into the development of these tumors, and dysregulated cell signaling pathways have been identified that are now the focus of specific molecularly targeted therapies (Appendices 1 and 2). 1,4,18,21,26,29,53,54,56,60 The epidermal growth factor receptors, which are part of a family of tyrosine kinase growth factor receptors named Her, are cell surface transmembrane proteins important in cell growth and proliferation as well as cell survival, motility, and resistance to chemotherapy and radiation therapy. In GBMs, EGFR is both overexpressed and truncated in the ectodomain, giving rise to a ligand-independent, constitutively activated form called EGFRviii. ${ }^{48,54}$ The EGFRviii strongly and persistent- 
ly activates the PI3K survival antiapoptotic pathway. ${ }^{18,53}$ The constitutive PI3K signaling that is driven by EGFRviii results in what has been termed "pathway addiction." 78 By promoting chronic dependence on the PI3K pathway, interruption of the dysregulated pathway (that is, by EGFR inhibitors) results in tumor cell death.

Nevertheless, despite the fact that nearly $40 \%$ of GBMs express EGFRviii, tyrosine kinase inhibitors (one class of EGFR inhibitors) such as gefitinib and erlotinib have had very modest therapeutic efficacy $(10-15 \%$ response rates). ${ }^{56,60}$ Limited response to EGFR inhibition in GBM appears to reflect dysregulation in downstream signaling in the PI3K pathway. Phosphatase and tensin homolog, also referred to as PTEN, is a phosphatase that inhibits PI3K signaling and that is commonly lost in GBMs. ${ }^{4,18,53}$ Without PTEN, the PI3K pathway is constitutively active and independent of EGFR signaling. Mellinghoff, et al. ${ }^{54}$ elegantly demonstrated that response to EGFR tyrosine kinase inhibitors in GBM is dependent on coexpression of both EGFRviii and PTEN. Loss of PTEN in GBMs (seen in $50 \%$ of cases) results in resistance to EGFR inhibitors.

Based on these data, we would suggest that there are several important aspects to targeted therapy. First, single-agent molecule inhibitors are not likely to be effective because GBM, like most epithelial cancers, has multiple interactive and dysregulated cell signaling pathways that will require multipoint combinatorial targeted therapies for effective tumor cell killing. Second, GBM may be stratified into molecular subtypes, predicting response to EGFR inhibitors (EGFRviii and PTEN coexpression) or lack thereof. Third, in patients with GBMs expressing EGFRviii and with loss of PTEN, the inhibition of the PI3K pathway downstream (that is, at Akt or mammalian target of rapamycin) may promote tumor responsiveness to EGFR inhibition. ${ }^{33}$ Finally, based on the results of these studies, we suggest that molecular profiling of GBM (an evolving and increasingly complicated area of research) is likely to provide insight into rational targeted therapy. Two recent examples of combinatorial regimens in which a targeted therapy and cytotoxic chemotherapy were used for recurrent GBM are seen in Table 1, whereas more recent trials in which standard chemotherapies were used are detailed in Table 2. The evolving role of immunotherapy and, in particular, the role of dendritic cell vaccines for the treatment of GBM is discussed elsewhere in this issue.

Although it is used infrequently in the majority of neurooncology centers, resection of the recurrent GBM followed by Gliasite implantation into the surgical cavity is useful in certain highly selected patients. ${ }^{19}$ Gliasite is a balloon catheter device permitting postsurgical instillation with a liquid iodine-131 radiopharmaceutical agent, Iotrex..$^{74}$ This device offers the newest method of delivery for brachytherapy. Deciding whom to treat with Gliasite is difficult, and the outcome is similar to that seen with many salvage chemotherapy protocols. Additionally, interpretation of magnetic resonance imaging studies following treatment is complicated (as is true for all local therapies) and often requires correlative metabolic imaging. A therapy similar to Gliasite (although it is noninvasive), fractionated stereotactic radiotherapy for recurrent GBM is advocated at some neurooncology centers. As an example of outcomes, a recent European report of 59 patients with recurrent GBM related a median progression-free survival of 5 months and overall survival period of 8 months. ${ }^{75}$

Two other novel approaches for treating recurrent GBM include attempts to modify MGMT expression in tumors, either by depleting it using protracted TMZ dosing (socalled metronomic therapy) or by providing an alternative MGMT substrate such as $\mathrm{O}^{6}$-benzylguanine, which inactivates MGMT. ${ }^{13,27,28,30,80}$ Prolonged TMZ dosing not only results in an increased exposure to the drug, but also will deplete MGMT by suicide inactivation, a direct consequence of the MGMT enzyme repairing a single TMZ-induced DNA methyl adduct. ${ }^{30}$ This is an unproven hypothesis at this time, but it has been embraced at a number of neurooncology centers and will probably enter trials this year. An alternative strategy uses imidazotetrazinone-methylating agents such as $\mathrm{O}^{6}$-benzylguanine. ${ }^{27,30,80}$ Unfortunately, trials using this strategy have found myelosuppression to be dose-limiting, and consequently, tolerable doses of TMZ (or $\mathrm{BCNU}$ ) have been considerably less than those achieved without concurrent $\mathrm{O}^{6}$-benzylguanine administration. Most important, response rates and survival times have been disappointing, probably due to the need to decrease the alkylator-based chemotherapy dose.

In the following section we briefly summarize ongoing clinical trials for patients with GBM in four of the major US brain tumor consortia. This list is not reflective of all trials available for GBM because there are numerous single-institution trials for patients with both initially diagnosed and recurrent GBM.

\section{Brain Tumor Consortia Trials}

\section{New Approaches to Brain Tumor Therapy}

Adjuvant Therapy. Four protocols are presently open for newly diagnosed GBM. These include the following: 1) neoadjuvant oxaliplatin followed by the EORTC/NCIC regimen; 2) talampanel given with the EORTC/NCIC regi-

TABLE 1

Combinatorial Phase II trials for recurrent GBM*

\begin{tabular}{|c|c|c|c|c|c|c|}
\hline \multirow[b]{2}{*}{ Authors \& Year } & \multirow[b]{2}{*}{ No. of Patients } & \multirow[b]{2}{*}{ Regimen } & \multicolumn{4}{|c|}{ Outcome $(\%)$} \\
\hline & & & $\begin{array}{l}\text { Response } \\
\text { Rate }\end{array}$ & $\begin{array}{l}\text { Stable } \\
\text { Disease }\end{array}$ & $\begin{array}{l}\text { 6-Mo } \\
\text { PFS }\end{array}$ & $\begin{array}{l}\mathrm{mOS} \\
(\mathrm{wks})\end{array}$ \\
\hline Dresemann, 2003 & 30 & hydroxyurea + Gleevec & 17 & 37 & 32 & NS \\
\hline Reardon, et al., 2005 & 33 & hydroxyurea + Gleevec & 9 & 42 & 27 & 14.4 \\
\hline Stark-Vance, 2005 & $21(11 \mathrm{w} / \mathrm{GBM})$ & Avastin + CPT-11 & 43 & 52 & NS & NS \\
\hline
\end{tabular}

$*$ mOS $=$ median overall survival; NS = not stated PFS = progression-free survival. 
TABLE 2

Phase II trials for recurrent $G B M^{*}$

\begin{tabular}{|c|c|c|c|c|c|c|}
\hline \multirow[b]{2}{*}{ Authors \& Year } & \multirow[b]{2}{*}{ Regimen } & \multirow[b]{2}{*}{$\begin{array}{l}\text { No. of } \\
\text { Patients }\end{array}$} & \multirow[b]{2}{*}{$\begin{array}{l}\text { \% Chemo- } \\
\text { Naïve }\end{array}$} & \multicolumn{2}{|c|}{ Outcome $(\%)$} & \multirow[b]{2}{*}{ mOS (wks) } \\
\hline & & & & $\begin{array}{l}\text { Response } \\
\text { Rate }\end{array}$ & 6-Mo PFS & \\
\hline Yung, 2000 & TMZ & 112 & 35 & 5 & 21 & NR \\
\hline Brada, et al., 2001 & TMZ & 138 & 71 & 8 & 18 & 23 \\
\hline Kapelle, et al., 2001 & PCV & 82 & 8 & 11 & 29 & 35 \\
\hline Wong, et al., 2001 & 8 trials & 225 & 20 & 6 & 15 & 25 \\
\hline Brandes, et al., 2002 & TMZ & 42 & 0 & 19 & 24 & 30 \\
\hline Groves, et al., 2002 & $\mathrm{TMZ}+$ marimastat & 44 & 43 & 14 & 39 & 45 \\
\hline Jaeckle, et al., 2003 & $\mathrm{TMZ}+\mathrm{cRA}$ & 40 & 28 & 5 & 32 & 35 \\
\hline Brandes, et al., 2003 & $\mathrm{BCNU}$ & 40 & 100 & 15 & 17.5 & 30 \\
\hline Brandes, et al., 2004† & TMZ + CDDP & 50 & 100 & 20 & 34 & 48 \\
\hline Brandes, et al., 2004 & CPT-11 + BCNU & 42 & 0 & 21 & 30 & 50 \\
\hline See, et al., 2004 & cRA & 85 & 0 & 4 & 19 & 10 \\
\hline Chamberlain, et al., 2004 & CYC & 40 & 0 & 17.5 & 20 & 32 \\
\hline
\end{tabular}

$* \mathrm{CDDP}=$ cisplatin; chemo $=$ chemotherapy; $\mathrm{cRA}=$ cis-retinoic acid $\mathrm{CYC}=$ cyclophosphamide NR $=$ not reported PCV $=$ procarbazine, CCNU (lomustine), and vincristine.

$\dagger$ Reference 8 .

$\$$ Reference 9 .

men; 3) EMD 121974 (cilengitide) given with the EORTC/ NCIC regimen; and 4) poly-ICLC given after concurrent TMZ and radiation therapy and with TMZ given after radiation therapy.

In neoadjuvant oxaliplatin therapy, oxaliplatin is administered intravenously prior to concurrent radiotherapy and TMZ in an attempt to define activity for this platinoid salt. Talampanel, an oral agent studied initially at the National Cancer Institute, is a glutaminergic antagonist originally designed as an anticonvulsant agent that was found to inhibit glioma cell motility. The agent EMD is an intravenous integrin inhibitor $\left(\alpha_{5} \beta_{3}\right)$ with antiangiogenic activity. Last, intramuscular poly-ICLC has four purported mechanisms of action, including interferon induction, immune modulation, antiproliferative antiapoptotic activity, and regulation of a variety of genes.

Salvage Therapy. Four trials are presently open for recurrent GBM. These include the following: 1) SDX-102; 2) Bay 43-9006 (sorafenib); 3) interstitial ${ }^{131}$ I-chTNT-1/B (Cotara; Peregrine Pharmaceuticals, Inc., Tustin, CA); and 4) high-dose methotrexate using an intratumoral microdialysis catheter. The SDX molecule is a novel purine antagonist that is also administered intravenously and requires tumor tissue screening for the presence or absence of the repair enzyme methylthioadenosine phosphorylase. If this enzyme is absent, patients are considered candidates for SDX-102 therapy. Sorafenib, recently approved for renal cell carcinoma, is an oral anti-Raf inhibitor with antiangiogenic activity. Cotara, an antihistone monoclonal antibody directed at necrotic tumor and conjugated to iodine-131, is administered by CED in a dose-finding study. Last, high-dose methotrexate is studied pharmacologically by using intratumoral microdialysis catheters, and intratumoral methotrexate levels are compared with those in serum.

\section{North American Brain Tumor Consortia}

Adjuvant Therapy. One trial is open for newly diagnosed GBM, as follows: R115777 (tipifarnib) and radiotherapy.
Tipifarnib is a farnesyl inhibitor that blocks the Ras signaling pathway.

Salvage Therapy. Three trials are open for recurrent GBM; these include the following: 1) EMD 121974 (cilengitide); 2) depsipeptide; 3) OSI-774 (erlotinib; Tarceva) and CCI-779 (temsirolimus); and 4) SAHA and TMZ.

Cilengitide, as discussed earlier, is an integrin inhibitor with antiangiogenic activity. Depsipeptide, like SAHA, is an HDAC inhibitor. Erlotinib combined with temsirolimus is a rational combination of small-molecule inhibitors designed to block both EGFR and downstream elements (such as mammalian target of rapamycin) of the PI3K/Akt survival pathway. A hydroxamic acid derivative, SAHA is one of several HDAC inhibitors. The HDAC molecule functions as a transcriptional repressor by compaction of chromatin, and HDAC inhibition causes cell cycle arrest, apoptosis, and differentiation.

\section{North Central Cancer Treatment Group}

Adjuvant Therapy. Two trials are open for newly diagnosed GBM. These include the following: 1) radiotherapy and the combination of CPT-11 and BCNU; and 2) OSI774 (erlotinib; Tarceva) in conjunction with the EORTC/ NCIC regimen. The agent CPT-11 is a topoisomerase 1 inhibitor with activity against recurrent GBM (Table 3), and may be synergistic when administered with BCNU. Tarceva, as mentioned earlier, is a tyrosine kinase inhibitor that blocks EGFR activation.

Salvage Therapy. A single salvage trial is open, and it involves SAHA, a hydroxamic acid derivative and one of several HDAC inhibitors, as discussed earlier.

\section{Radiation Treatment Oncology Group}

Adjuvant Therapy. A single trial is open, in which the EORTC/NCIC regimen is being compared with a dosedense TMZ regimen.

The value of increasing the TMZ dose in the treatment of 
TABLE 3

The CPT-11 regimen for recurrent GBM

\begin{tabular}{lccrr}
\hline \hline \multirow{2}{*}{ Authors \& Year } & Chemo Schedule & No. of Patients & Response Rate & Outcome (\%) \\
\cline { 3 - 5 } Friedman, et al., 1999 & weekly $\times 4$ & 48 & 17 & NS \\
Chamberlain, 2002 & every 3 wks & 40 & 6 & 6.0 \\
Raymond, et al., 2003 & every 3 wks & 27 & 2.2 & 43 \\
Batchelor, et al., 2004 & weekly $\times 4$ & 18 & 9 & 36 \\
Prados, et al., 2004 & every 3 wks & 42 & 0 & 8.3 \\
\hline
\end{tabular}

GBM is still controversial. This large randomized trial will not only enable validation of the EORTC/NCIC results (standard-dose TMZ), but in addition, the investigators will attempt to prove the principle that increasing TMZ (that is, by using the 21 days on and 7 days off schedule, with TMZ given at $100 \mathrm{mg} / \mathrm{m}^{2} /$ day) positively affects the outcome in newly treated patients with GBM. Furthermore, the protocol allows patients older than 69 years of age to be treated, and, in patients with responsive tumors, treatment can extend beyond the 6-month postradiation TMZ regimen.

Salvage Therapy. No trial is available at this time.

\section{Conclusions}

The treatment of adults with GBM continues to evolve. The EORTC/NCIC regimen now serves as the standard initial therapy with which other trials will be compared. The next development in the adjuvant treatment of GBM is likely to be the addition of a targeted therapy to TMZ administration, as is reflected in the clinical trials conducted by the brain tumor consortia. These targeted therapies may be CED-based or small-molecule inhibitors, depending on the results of ongoing trials. The EORTC/NCIC investigators, in conjunction with the RTOG, are planning a follow-up randomized Phase III trial for adjuvant treatment of GBM, and will compare extended TMZ treatment (from 6 to 12 months after radiotherapy) with a dose-dense TMZ schedule. Additionally, the pharmaceutical industry is planning new randomized trials for recurrent GBM, in which a variety of new compounds such as enzastaurin (Eli Lilly Pharmaceuticals, Indianapolis, IN), a novel protein kinase $\mathrm{C}$ inhibitor, will be compared with the best standard chemotherapy.

New targeted therapies will be increasingly used for recurrent GBM in Phase I and II trials, and are likely to provide new directions for adjuvant treatment. A novel concept, that of gliomas as neural stem cell-derived tumors, may stimulate the design of new therapies directed at these pluripotent precursor cells with their purportedly distinct cell surface markers (for example, CD133). ${ }^{63}$ Finally, GBMs will be increasingly subjected to molecular profiling, which will permit stratification of patients into those who are likely to respond to alkylator-based chemotherapy and those who are not likely to respond. The latter group of patients would be offered enrollment in alternative and investigational trials both initially and at recurrence.

\section{Appendix 1}

Molecular biology of GBMs* overexpression of EGFR constitutive activation of EGFRviii (no ectodomain) mutations in $40 \%$ of GBMs

overactivation of PI3K-Akt survival pathway

upregulation of antiapoptotic factors, promotion of cell survival

PTEN mutations (negative regulator PI3K) in $45 \%$ of GBMs mutations in tumor suppressor gene $p 53$

cell cycle control, DNA repair, induction of apoptosis

p53 mutated in $30 \%$ of GBMs

overexpression of PDGFR

activation of angiogenesis, PI3K, Ras-MAPK

mutations in $25 \%$ of GBMs

*MAPK = mitogen-activated protein kinase; PDGFR = platelet-derived growth factor receptor.

\section{Appendix 2}

Targeted therapy for GBM

EGFR tyrosine kinase inhibitors

gefitinib (Iressa)

erlotinib (Tarceva)

imatinib (Gleevec)

PI3K inhibitors

wortmannin

LY294002

mammalian target of rapamycin inhibitors

rapamycin

CCI-779

RAD001

farnesyl transferase inhibitors

tipifarnib (R115777)

lonafarnib (SCH66336)

antiangiogenic agents

imids

CC-5103

PTK787

antiinvasive agents

cilengitide

marimastat

cell growth \& migration inhibitor

cis-retinoic acid (Accutane)

\section{Appendix 3}

Intratumoral drug delivery

direct injection (for example, DTI-101)

intracavitary administration (for example, ${ }^{131} \mathrm{I}-\mathrm{TM}-601$ )

chronic low-flow microinfusion

controlled-release polymer implants (for example, Gliadel wafers) CED

\section{Appendix 4}

Targeted toxins for CED of drugs*

TP-38

TGF- $\alpha$ (EGFR ligand)

linked to pseudomonas exotoxin (termed PE-38) 


\author{
Tf-CRM107 (TransMID; Xenova Biomedix Pharmaceuticals) \\ transferrin \\ linked to diphtheria toxin \\ IL13-PE38QQR (NeoPharm, Inc.) \\ IL-13 \\ linked to pseudomonas exotoxin \\ IL4-PE38KDEL \\ IL-4 \\ linked to pseudomonas exotoxin \\ ${ }^{131}$ I-chTNT-1/B (Cotara; Peregrine Pharmaceuticals, Inc.) \\ monoclonal antibody to histone protein \\ linked to iodine-131 \\ $*$ TGF- $\alpha$ transforming growth factor- $\alpha$
}

\section{References}

1. Aldape KD, Ballman K, Furth A, et al: Immunohistochemical detection of EGFRvIII in high malignancy grade astrocytomas and evaluation of prognostic significance. J Neuropathol Exp Neurol 63:700-707, 2004

2. Ataman F, Poortmans P, Stupp R, et al: Quality assurance of the EORTC 26981/22981; NCIC CE3 intergroup trial on radiotherapy with or without temozolomide for newly-diagnosed glioblastoma multiforme: the individual case review. Eur J Cancer 40:1724-1730, 2004

3. Batchelor TT, Gilbert MR, Supko JG, et al: Phase 2 study of weekly irinotecan in adults with recurrent malignant glioma: final report of NABTT 97-11. Neurooncol 6:21-27, 2004

4. Bianco R, Shin I, Ritter CA, et al: Loss of PTEN/MMAC1/TEP in EGF receptor-expressing tumor cells counteracts the antitumor action of EGFR tyrosine kinase inhibitors. Oncogene 22: 2812-2822, 2003

5. Bobo RH, Laske DW, Akbasak A, et al: Convection-enhanced delivery to macromolecules in the brain. Proc Natl Acad Sci U S A 91:2076-2080, 1994

6. Brada M, Hoang-Xuan K, Rampling R, et al: Multicenter phase II trial of temozolomide in patients with glioblastoma multiforme at first relapse. Ann Oncol 12:259-266, 2001

7. Brandes AA, Ermani M, Basso U, et al: Temozolomide in patients with glioblastoma at second relapse after first line nitrosoureaprocarbazine failure: a phase II study. Oncology 63:38-41, 2002

8. Brandes AA, Tosoni A, Amistà P, et al: How effective is BCNU in recurrent glioblastoma in the modern era? A phase II trial. Neurology 63:1281-1284, 2004

9. Brandes AA, Tosoni A, Basso U, et al: Second-line chemotherapy with irinotecan plus carmustine in glioblastoma recurrent or progressive after first-line temozolomide chemotherapy: a phase II study of the Gruppo Italiano Cooperativo di NeuroOncologia (GICNO). J Clin Oncol 22:4779-4786, 2004

10. Brandes AA, Vastola F, Basso U, et al: A prospective study on glioblastoma in the elderly. Cancer 97:657-662, 2003

11. Brem H, Mahaley MS Jr, Vick NA, et al: Interstitial chemotherapy with drug polymer implants for the treatment of recurrent gliomas. J Neurosurg 74:441-446, 1991

12. Brem H, Piantadosi S, Burger PC, et al: Placebo-controlled trial of safety and efficacy of intraoperative controlled delivery by biodegradable polymers of chemotherapy for recurrent gliomas. The Polymer-Brain Tumor Treatment Group. Lancet 345: 1008-1012, 1995

13. Chamberlain MC: Salvage chemotherapy with CPT-11 for recurrent glioblastoma multiforme. J Neurooncol 56:183-188, 2002

14. Chamberlain MC, Tsao-Wei DD, Groshen S: Salvage chemotherapy with cyclophosphamide for recurrent temozolomiderefractory anaplastic astrocytoma. Cancer 106:172-179, 2006

15. Chan JL, Lee SW, Fraass BA, et al: Survival and failure patterns of high-grade gliomas after three-dimensional conformal radiotherapy. J Clin Oncol 20:1635-1642, 2002

16. Chang SM, Parney IF, Huang W, et al: Patterns of care for adults with newly diagnosed malignant glioma. JAMA 293:557-564, 2005
17. Chen MY, Lonser RR, Morrison PF, et al: Variables affecting convection-enhanced delivery to the striatum: a systematic examination of rate of infusion, cannula size, infusate concentration, and tissue-cannula sealing time. J Neurosurg 90:315-320, 1999

18. Choe G, Horvath S, Cloughesy TF, et al: Analysis of the phosphatidylinositol 3'-kinase signaling pathway in glioblastoma patients in vivo. Cancer Res 63:2742-2746, 2003

19. Combs SE, Thilmann C, Edler L, et al: Efficacy of fractionated stereotactic reirradiation in recurrent gliomas: long-term results in 172 patients treated in a single institution. J Clin Oncol 23: 8863-8869, 2005

20. Curran WJ Jr, Scott CB, Horton J, et al: Recursive partitioning analysis of prognostic factors in three Radiation Therapy Oncology Group malignant glioma trials. J Natl Cancer Inst 85: 704-710, 1993

21. Dancey JE, Freidlin B: Targeting epidermal growth factor receptor-are we missing the mark? Lancet 362:62-64, 2003

22. DeAngelis LM: Brain tumors. N Engl J Med 344:114-123, 2001

23. Debinski W: Local treatment of brain tumors with targeted chimera cytotoxic proteins. Cancer Invest 20:801-809, 2002

24. Dresemann G: STI 571/hydroxyurea in progressive, pretreated glioblastoma (GB) patients (pts.). Proc Am Soc Clin Oncol 22:116, 2003 (Abstract)

25. Fine HA, Dear KBG, Loeffler JS, et al: Meta-analysis of radiation therapy with and without adjuvant chemotherapy for malignant gliomas in adults. Cancer 71:2585-2597, 1993

26. Friedman HS, Bigner DD: Glioblastoma multiforme and the epidermal growth factor receptor. N Engl J Med 353:1997-1999, 2005

27. Friedman HS, Kokkinakis DM, Pluda J, et al: Phase I trial of $\mathrm{O}^{6}$ benzylguanine for patients undergoing surgery for malignant glioma. J Clin Oncol 16:3570-3575, 1998

28. Friedman HS, Petros WP, Friedman AH, et al: Irinotecan therapy in adults with recurrent or progressive malignant glioma. J Clin Oncol 17:1516-1525, 1999

29. Galanis E, Buckner JC, Maurer MJ, et al: Phase II trial of Temsirolimus (CCI-779) in recurrent glioblastoma multiforme: a North Central Cancer Treatment Group study. J Clin Oncol 23:5294-5304, 2005

30. Gerson SL: Clinical relevance of $M G M T$ in the treatment of cancer. J Clin Oncol 20:2388-2399, 2002

31. Glantz M, Chamberlain M, Liu Q, et al: Temozolomide as an alternative to irradiation for elderly patients with newly diagnosed malignant gliomas. Cancer 97:2262-2266, 2003

32. Glantz MJ, Cole BF, Forsyth PA, et al: Practice parameter: anticonvulsant prophylaxis in patients with newly diagnosed brain tumors. Report of the quality standards subcommittee of the American Academy of Neurology. Neurology 54:1886-1893, 2000

33. Goudar RK, Shi Q, Hjelmeland MD, et al: Combination therapy of inhibitors of epidermal growth factor receptor/vascular endothelial growth factor receptor 2 (AEE788) and the mammalian target of rapamycin (RAD001) offers improved glioblastoma tumor growth inhibition. Mol Cancer Ther 4:101-112, 2005

34. Grossman SA, O'Neill A, Grunnet M, et al: Phase III study comparing three cycles of infusional carmustine and cisplatin followed by radiation therapy with radiation therapy and concurrent carmustine in patients with newly diagnosed supratentorial glioblastoma multiforme: Eastern Cooperative Oncology Group trial 2394. J Clin Oncol 21:1485-1491, 2003

35. Grossman SA, Reinhard C, Colvin OM, et al: The intracerebral distribution of BCNU delivered by surgically implanted biodegradable polymers. J Neurosurg 76:640-647, 1992

36. Grossman SA, Wharam M, Sheidler V, et al: Phase II study of continuous infusion carmustine and cisplatin followed by cranial irradiation in adults with newly diagnosed high-grade astrocytoma. J Clin Oncol 15:2596-2603, 1997

37. Groves MD, Puduvalli VK, Hess KR, et al: Phase II trial of te- 
mozolomide plus the matrix metalloproteinase inhibitor, marimastat, in recurrent progressive glioblastoma multiforme. J Clin Oncol 20:1383-1388, 2002

38. Hegi ME, Diserens AC, Gorlia T, et al: $M G M T$ gene silencing and benefit from temozolomide in glioblastoma. N Engl J Med 352:997-1003, 2005

39. Hockaday DC, Shen S, Fiveash J, et al: Imaging glioma extent with 131I-TM-601. J Nucl Med 46:580-586, 2005

40. Jaeckle KA, Eyre HJ, Townsend JJ, et al: Correlation of tumor O6 methylguanine-DNA methyltransferase levels with survival of malignant astrocytoma patients treated with bis-chloroethylnitrosourea: a Southwest Oncology Group study. J Clin Oncol 16:3310-3315, 1998

41. Jaeckle KA, Hess KR, Yung WKA, et al: Phase II evaluation of temozolomide and 13-cis-retinoic acid for the treatment of recurrent and progressive malignant glioma: a North American Brain Tumor Consortium study. J Clin Oncol 21:2305-2311, 2003

42. Kappelle AC, Postma TJ, Taphoorn MJ, et al: PCV chemotherapy for recurrent glioblastoma multiforme. Neurology 56:118-120, 2001

43. Keles GE, Anderson B, Berger MS: The effect of extent of resection on time to tumor progression and survival in patients with glioblastoma multiforme of the cerebral hemisphere. Surg Neurol 52:371-379, 1999

44. Khuntia D, Mehta M: Motexafin gadolinium: a clinical review of a novel radioenhancer for brain tumors. Expert Rev Anticancer Ther 4:981-989, 2004

45. Kioi M, Kawakami K, Puri RK: Analysis of antitumor activity of an interleukin-13 (IL-13) receptor-targeted cytotoxin composed of IL-13 antagonist and Pseudomonas exotoxin. Clin Cancer Res 10:6231-6238, 2004

46. Kleinberg L, Grossman SA, Carson K, et al: Survival of patients with newly diagnosed glioblastoma multiforme treated with RSR13 and radiotherapy: results of a phase II New Approaches to Brain Tumor Therapy CNS Consortium safety and efficacy study. J Clin Oncol 20:3149-3155, 2002

47. Kowalczuk A, Macdonald RL, Amidei C, et al: Quantitative imaging study of extent of surgical resection and prognosis of malignant astrocytomas. Neurosurgery 41:1028-1038, 1997

48. Kuan CT, Wikstrand CJ, Bigner DD: EGF mutant receptor vIII as a molecule target in cancer therapy. Endocr Relat Cancer 8:83-96, 2001

49. Lacroix M, Abi-Said D, Fourney DR, et al: A multivariate analysis of 416 patients with glioblastoma multiforme: prognosis, extent of resection, and survival. J Neurosurg 95:190-198, 2001

50. Laperriere NJ, Leung PMK, McKenzie S, et al: Randomized study of brachytherapy in the initial management of patients with malignant astrocytoma. Int J Radiat Oncol Biol Phys 41: 1005-1011, 1998

51. Laske DW, Youle RJ, Oldfield EH: Tumor regression with regional distribution of the targeted toxin TF-CRM107 in patients with malignant brain tumors. Nat Med 3:1362-1368, 1997

52. Laws ER, Parney IF, Huang W, et al: Survival following surgery and prognostic factors for recently diagnosed malignant glioma: data from the Glioma Outcomes Project. J Neurosurg 99:467-473, 2003

53. Li B, Yuan M, Kim IA, et al: Mutant epidermal growth factor receptor displays increased signaling through the phosphatidylinositol-3 kinase/AKT pathway and promotes radioresistance in cells of astrocytic origin. Oncogene 23:4594-4602, 2004

54. Mellinghoff IK, Wang MY, Vivanco I, et al: Molecular determinants of the response of glioblastomas to EGFR kinase inhibitors. N Engl J Med 353:2012-2024, 2005

55. Nguyen TT, Pannu YS, Sung C, et al: Convective distribution of macromolecules in the primate brain demonstrated using computerized tomography and magnetic resonance imaging. J Neurosurg 98:584-590, 2003

56. Prados M, Chang S, Burton E, et al: Phase I study of OSI-774 alone or with temozolomide in patients with malignant glioma. Proc Am Soc Clin Oncol 22:99, 2003 (Abstract No. 394)

57. Prados MD, Yung WKA, Jaeckle KA, et al: Phase 1 trial of irinotecan (CPT-11) in patients with recurrent malignant glioma: a North American Brain Tumor Consortium study. Neuro-oncol 6:44-54, 2004

58. Raymond E, Fabbro M, Boige V, et al: Multicentre phase II study and pharmacokinetic analysis of irinotecan in chemotherapynaïve patients with glioblastoma. Ann Oncol 14:603-614, 2003

59. Reardon DA, Egorin MJ, Quinn JA, et al: Phase II study of imatinib mesylate plus hydroxyurea in adults with recurrent glioblastoma multiforme. J Clin Oncol 23:9359-9368, 2005

60. Rich JN, Reardon DA, Peery T, et al: Phase II trial of gefitinib in recurrent gliolblastoma. J Clin Oncol 22:133-142, 2004

61. Ries LAG, Eisner MP, Kosary CL, et al (eds): SEER Cancer Statistics Review, 1975-2002. Bethesda, MD: National Cancer Institute, 2005 (http://seer.cancer.gov/csr/1975_2002/) [Accessed 1 March 2006]

62. Roa W, Brasher PMA, Bauman G, et al: Abbreviated course of radiation therapy in older patients with glioblastoma multiforme: a prospective randomized clinical trial. J Clin Oncol 22: 1583-1588, 2004

63. Sanai N, Alvarez-Buyulla A, Berger MS: Neural stem cells and the origin of gliomas. N Engl J Med 353:811-822, 2005

64. See SJ, Levin VA, Yung WKA, et al: 13-cis-retinoic acid in the treatment of recurrent glioblastoma multiforme. Neuro-oncol 6:253-258, 2004

65. Selker RG, Shapiro WR, Burger P, et al: The Brain Tumor Cooperative Group NIH Trial 87-01: a randomized comparison of surgery, external radiotherapy, and carmustine versus surgery, interstitial radiotherapy boost, external radiation therapy, and carmustine. Neurosurgery 51:343-357, 2002

66. Shinoda J, Sakai N, Murase S, et al: Selection of eligible patients with supratentorial glioblastoma multiforme for gross total resection. J Neurooncol 52:161-171, 2001

67. Simpson JR, Horton J, Scott C, et al: Influence of location and extent of surgical resection on survival of patients with glioblastoma multiforme: results of three consecutive Radiation Therapy Oncology Group (RTOG) clinical trials. Int J Rad Oncol Biol Phys 26:239-244, 1993

68. Souhami L, Seiferheld W, Brachman D, et al: Randomized comparison of stereotactic radiosurgery followed by conventional radiotherapy with carmustine to conventional radiotherapy with carmustine for patients with glioblastoma multiforme: report of Radiation Therapy Oncology Group 93-05 protocol. Int J Radiat Oncol Biol Phys 60:853-860, 2004

69. Stark-Vance V: Bevacizumab and CPT-11 in the treatment of relapsed malignant glioma. Neuro-Oncol 7:369, 2005 (Abstract)

70. Stewart LA: Glioma Meta-analysis Trialists (GMT) Group: Chemotherapy in adult high-grade glioma: a systematic review and meta-analysis of individual data from 12 randomised trials. Lancet 359:1011-1018, 2002

71. Stupp R, Dietrich PY, Ostermann Kraljevic S, et al: Promising survival for patients with newly diagnosed glioblastoma multiforme treated with concomitant radiation plus temozolomide followed by adjuvant temozolomide. J Clin Oncol 20:1375-1382, 2002

72. Stupp R, Mason WP, van den Bent M, et al: Radiotherapy plus concomitant and adjuvant temozolomide for glioblastoma. $\mathbf{N}$ Engl J Med 352:987-996, 2005

73. Taphoorn MJB, Stupp R, Coens C, et al: Health-related quality of life in patients with glioblastoma: a randomised controlled trial. Lancet Oncol 6:937-944, 2005

74. Tatter SB, Shaw EG, Rosenblum ML, et al: An inflatable balloon catheter and liquid ${ }^{125}$ I radiation source (GliaSite Radiation Therapy System) for treatment of recurrent malignant glioma: multicenter safety and feasibility trial. J Neurosurg 99:297-303, 2003

75. Tolcher AW, Gerson SL, Denis L, et al: Marked inactivation of $\mathrm{O}^{6}$ - alkylguanine-DNA alkyltransferase activity with protracted temozolomide schedules. Br J Cancer 88: 1004-1011, 2003 
76. Walker MD, Alexander E, Hunt WE, et al: Evaluation of BCNU and/or radiotherapy in the treatment of anaplastic gliomas. A cooperative clinical trial. J Neurosurg 49:333-343, 1978

77. Walker MD, Green SB, Byar DP, et al: Randomized comparisons of radiotherapy and nitrosoureas for the treatment of malignant gliomas after surgery. N Engl J Med 303: 1323-1329, 1980

78. Weinstein IB: Cancer: Enhanced: Addiction to oncogenes-the Achilles heal of cancer. Science 297:63-64, 2002

79. Westphal M, Hilt DC, Bortey E, et al: A phase 3 trial of local chemotherapy with biodegradable carmustine (BCNU) wafers (Gliadel wafers) in patients with primary malignant glioma. Neuro-oncol 5:79-88, 2003

80. Wick W, Steinbach JP, Küker WM, et al: One week on/one week off: a novel active regimen of temozolomide for recurrent glioblastoma. Neurology 62:2113-2115, 2004
81. Wong ET, Hess KR, Gleason MJ, et al: Outcomes and prognostic factors in recurrent glioma patients enrolled onto phase II clinical trials. J Clin Oncol 17:2572-2578, 1999

82. Yung WK: Temozolomide in malignant gliomas. Semin Oncol 27 (3 Suppl):27-34, 2000

Manuscript received January 23, 2006.

Accepted in final form March 3, 2006.

Address reprint requests to: Marc C. Chamberlain, M.D., Department of Interdisciplinary Oncology, Moffitt Cancer Center and Research Institute, 12902 Magnolia Avenue, Tampa, Florida 33612-0804. email: ChambeMC@moffitt.usf.edu. 